\title{
Semi-supervised learning of a nonnative phonetic contrast: How much feedback is enough?
}

\author{
Beverly A. Wright ${ }^{1} \cdot$ Emma K. LeBlanc $^{2} \cdot$ David F. Little $^{3} \cdot$ Jessica S. Conderman ${ }^{2} \cdot$ Courtney Coburn Glavin $^{2}$
}

Published online: 6 May 2019

(C) The Psychonomic Society, Inc. 2019

\begin{abstract}
Semi-supervised learning refers to learning that occurs when feedback about performance is provided on only a subset of training trials. Algorithms for semi-supervised learning are popular in machine learning because of their minimal reliance on labeled data. There have been, however, only a few reports of semi-supervised learning in humans. Here we document human semi-supervised learning on a nonnative phonetic classification task. Classification performance remained unchanged when 60 feedback trials were provided on each of the two days of training. In contrast, performance improved when 60 feedback trials were combined with 240 no-feedback trials each day. In variants of this successful semi-supervised regimen, increasing the daily number of feedback trials from 60 to 240 did not increase the amount of learning, while decreasing that number to 30 abolished learning. Finally, replacing the no-feedback trials with stimulus exposure alone had little effect on the outcome. These results were an unexpected consequence of combining training periods with feedback and testing periods without feedback, illustrating that nofeedback testing can influence learning outcomes. More broadly, these data suggest that task performance with feedback can function as an all-or-none trigger for recruiting the contribution of trials without feedback, or mere stimulus exposures, to human learning.
\end{abstract}

Keywords Perceptual learning $\cdot$ Long-term memory $\cdot$ Speech perception

Learning can be divided into three broad categories in terms of the consistency with which feedback about performance is provided during training. Learning that occurs with trial-by-trial feedback is denoted supervised learning, while learning that occurs without any feedback is denoted unsupervised learning. Between these two extremes, learning that occurs when feedback is provided only on a subset of trials is denoted semi-supervised learning. Semi-supervised learning is important for both theory and application, because it indicates that when feedback is required for learning, it is not necessarily needed throughout the

Beverly A. Wright

b-wright@northwestern.edu

1 Department of Communication Sciences and Disorders, Knowles Hearing Center, Northwestern Institute for Neuroscience, Northwestern University, Evanston, IL, USA

2 Department of Communication Sciences and Disorders, Northwestern University, Evanston, IL, USA

3 Department of Electrical and Computer Engineering, Johns Hopkins University, Baltimore, MD, USA course of training. In principle, semi-supervised learning could occur for many tasks, because there are general-purpose algorithms for semi-supervised machine learning (Zhu \& Goldberg, 2009). However, the possibility that semi-supervised learning may occur in humans has received relatively little attention (e.g., Gibson, Rogers, \& Zhu, 2013). Here we describe a case of semi-supervised learning in humans that became apparent unexpectedly during the course of investigating a different question about learning on a speech-perception task.

\section{Semi-supervised learning}

We are aware of only a few previous reports of semisupervised learning in humans (e.g., Kalish, Rogers, Lang, \& Zhu, 2011; Kalish, Zhu, \& Rogers, 2015; Lake \& McClelland, 2011; Rogers, Kalish, Gibson, Harrison, \& Zhu, 2010; Vong, Navarro, \& Perfors, 2016; Zhu et al., 2010; Zhu, Rogers, Qian, \& Kalish, 2007). The premise of the majority of these investigations was that semi-supervised learning is demonstrated if trials without feedback 
(unsupervised trials) lead to changes in category boundary decisions about categories that were initially learned from trials with feedback (supervised trials). For example, Zhu et al. (2007) examined the classification of images of what were said to be particles of pollen. On each of the first 20 trials, one of two stimuli was presented - a relatively spikey stimulus or a relatively smooth stimulus. Participants were asked to label those stimuli as "Belianthus" or "Nortulaca" and were given feedback about their responses. They were then asked to label several hundred stimuli without feedback. These stimuli were distributed along a spikey-to-smooth continuum, but were selected from two Gaussian distributions with modes that were shifted toward the spikier stimuli for one group and toward the smoother stimuli for another group. After the feedback phase and again after the nofeedback phase, participants completed tests without feedback on 21 stimuli that were evenly distributed across the spikey-to-smooth continuum to determine the category boundary inferred from the training. Immediately after the feedback phase, participants placed the category boundary midway between the two labeled stimuli. However, after the no-feedback phase, the boundary shifted toward either the spikier or the smoother stimuli, moving in the same direction as the modes of the stimulus distributions during the no-feedback phase. The implication is that the trials without feedback altered the category boundary originally learned from the trials with feedback.

The final category boundaries in the report of Zhu et al. (2007) and in similar studies do not appear to be attributable to the influence of the no-feedback trials alone. For instance, in one investigation, two groups of participants were presented different stimulus sets for the initial trials with feedback, leading to different initial category boundaries, and then were presented a common, larger set of stimuli for the trials without feedback (Kalish et al., 2011). If the final category boundary were determined solely by the trials without feedback, that boundary should have been the same for both groups, but it was not. Instead, although the category boundary for each group shifted after the no-feedback phase, indicating semi-supervised learning, the final boundaries still differed between the groups. Furthermore, on the basis of estimates from modeling, trials with feedback were weighted more heavily than trials without feedback (Lake \& McClelland, 2011; Zhu et al., 2007).

\section{Present investigation}

The present investigation originally arose from two observations about learning on basic perceptual-discrimination tasks. First, this learning appears to require sufficient training within a day in order for performance to improve across days. For example, performance on an auditory frequencydiscrimination task improved across multiple daily training sessions with 900 training trials per day, but not with 360 trials per day (Wright \& Sabin, 2007). Likewise, learning occurred on a visual orientation-discrimination task with 400 trials per day, but not with 160 trials per day (Aberg, Tartaglia, \& Herzog, 2009). The outcomes were the same even when the total number of training trials was held constant across regimens by adjusting the number of days of training. Similar results have been reported for learning on a letterenumeration task (e.g., Hauptmann \& Karni, 2002; Hauptmann, Reinhart, Brandt, \& Karni, 2005). Second, once the amount of daily training is sufficient to induce across-day learning, additional daily training can be superfluous. For instance, increases in the number of training trials per day did not lead to greater learning on auditory temporal-interval discrimination (Wright \& Sabin, 2007), interaural timedifference discrimination (Ortiz \& Wright, 2010), or other nondeclarative tasks (e.g., mirror reading, Ofen-Noy, Dudai, \& Karni, 2003; or motor sequencing, Savion-Lemieux \& Penhune, 2005). Together these results suggest that trials within a day must integrate to a threshold in order to induce learning that persists across days, and that this learning threshold functions behaviorally as an all-or-none process.

Our initial plan had been to investigate whether the doseresponse properties of training on basic discrimination tasks using simple stimuli extended to the categorization of speech syllables. Toward this end, in the first experiment, we examined the influence of the daily training amount on learning of a nonnative phonetic contrast. The stimuli varied along a voiceonset-time (VOT) continuum. We had previously documented learning on this task that persisted across days using a regimen of 240 training trials per day with feedback (Wright, BaeseBerk, Marrone, \& Bradlow, 2015). We therefore systematically reduced the number of daily training trials with feedback from 240 , to 120 , to 60 , and to 30 across different groups of listeners and tracked the influence of that reduction on the magnitude of improvement.

The second experiment arose because we began to question whether trials without feedback that we had used to assess performance during daily pre- and posttraining tests might themselves be influencing learning. The training regimens in the first experiment included from 30 to 240 daily training trials with feedback (the focus of that experiment), but also 120-trial pre- and posttraining tests without feedback on each of the two days of training. We had initially worked under the assumption that these trials without feedback were essentially inert, on the basis of reports that learning on speechcategorization tasks using constant-stimulus techniques requires feedback (McCandliss, Fiez, Protopapas, Conway, \& McClelland, 2002; Tricomi, Delgado, McCandliss, McClelland, \& Fiez, 2006). In the second experiment, we tested that assumption. For one group of listeners, we removed all tests but the final one. For another group, we replaced all tests but the final one with stimulus exposure alone. 
The results (1) document semi-supervised learning on a speech-learning task, (2) indicate that this learning can be triggered by a small number of trials with feedback, (3) show that feedback on additional trials does not necessarily increase the amount of learning, and (4) suggest that task performance may not be required during the periods without feedback.

\section{Method}

The methods closely followed those of Wright et al. (2015).

\section{Listeners}

The listeners were 43 young adults. All were monolingual, native speakers of American English with normal hearing. They reported no history of language or learning disorders and did not consider themselves to be musicians. None had previous experience with psychoacoustic tasks. Listeners were recruited from the Northwestern University community and were either paid or received class credit for their participation. The data for the group who received 240 trials with feedback per day (see below) have been reported previously (Wright et al., 2015).

\section{Task and stimuli}

The task was phonetic classification of the initial consonant of consonant-vowel syllables. The consonant varied along a VOT continuum in $10-\mathrm{ms}$ steps from -70 to $+70 \mathrm{~ms}$. The continuum was divided into three categories: negative VOT $(<$ $\sim-25 \mathrm{~ms})$, labeled "mba," versus near-zero VOT ( $-25 \mathrm{~ms}$ to $\sim 25 \mathrm{~ms})$, labeled "ba," versus positive VOT $(>\sim+25$ $\mathrm{ms})$, labeled "pa." The "mba" label was phonetically inaccurate but was used because listeners could interpret it easily (see also Tremblay, Kraus, Carrell, \& McGee, 1997). A three-way contrast among negative, near-zero, and positive VOTs is present in Thai, Hindi, and many other languages. English includes the contrast between near-zero and positive VOTs, but not the contrast between negative and near-zero VOTs. However, native English speakers can acquire the three-way contrast with practice (McClaskey, Pisoni, \& Carrell, 1983; Pisoni, Aslin, Perey, \& Hennessy, 1982).

The stimuli were created using Praat software (Boersma, 2001) to vary the VOT in a token of "ba" and a token of "pa" spoken by a female native speaker of American English. They were presented to the left ear at a comfortable listening level over Sennheiser HD265 headphones. All listeners were tested in a double-walled, sound-attenuating booth.

\section{General protocol}

All listeners participated in two sessions on two consecutive days. Except where otherwise noted, each of the two sessions (both Days 1 and 2) included a familiarization phase, a pretraining test without feedback, a training phase with feedback, and a posttraining test without feedback. A hearing screening was conducted after the posttraining test on Day 2.

\section{Familiarization}

Each session began with a verbal description of the task followed by presentations of sample tokens from each of the three categories, with the correct response indicated for each token. The tokens were presented in random order, five times each, for a total of 15 presentations.

\section{Pre- and posttraining tests}

During each test, the 15 tokens along the VOT continuum were presented eight times each in random order, for a total of 120 trials. The task was to categorize each token. No feedback was provided.

\section{Training phase}

During the training phase, listeners practiced the categorization task with trial-by-trial feedback. The syllables were randomly selected from one of three normal distributions along the VOT continuum. Each distribution included five discrete, consecutive values of VOT. The distribution peaks were centered on $-50 \mathrm{~ms}$ for "mba" (distribution range: -70 to -30 $\mathrm{ms}$ ), $0 \mathrm{~ms}$ for "ba" (distribution range: -20 to $+20 \mathrm{~ms}$ ), and + $50 \mathrm{~ms}$ for "pa" (distribution range: +30 to $+70 \mathrm{~ms}$ ). Feedback on response accuracy was given after each trial via a visual display ("correct" or "incorrect"). Responses were considered correct if the VOT was between -70 to $-30 \mathrm{~ms}$ for "mba," 20 to $+20 \mathrm{~ms}$ for "ba," and + 30 to $+70 \mathrm{~ms}$ for "pa."

\section{Trained groups}

In the training-amount experiment, on each of two consecutive days, all listeners participated in a familiarization phase, a 120-trial pretraining test with no feedback, a training phase of 30-240 trials with feedback, and a 120-trial posttraining test with no feedback. Four listener groups differed in how many training trials with feedback they received per day: $30(n=7)$, $60(n=7), 120(n=8)$, or $240(n=7)$.

Two additional groups participated in the testing-influence experiment to evaluate the influence of the initial and intermediate testing periods on learning: a no-tests group and an exposure-only-tests group. The regimen of the no-tests group $(n=6)$ differed from that of the 60-trials-with-tests group (see 
above) only in that it did not include the pre- and posttraining tests on Day 1 or the pretraining test on Day 2. The regimen of the exposure-only-tests group $(n=8)$ differed from that of the 60-trials-with-tests group only in that the familiarization phases and all testing periods prior to the final posttraining test on Day 2 were replaced with stimulus exposure alone. During the periods of stimulus exposure alone, the same stimuli were presented as for the 60-trials-with-tests group, but no information was provided on the assigned response for each stimulus during the familiarization phases, and no response was required during the testing periods. Rather, listeners performed a written symbol-to-number matching task while the stimuli played in the background. For the self-paced matching task, listeners decoded a sequence of symbols into a sequence of numbers using a key. During the periods of stimulus exposure alone, the intersyllable interval was $\sim 5 \mathrm{~s}$, to approximate the average response time during the task performance periods. In sum, both of these groups practiced 60 trials with feedback per day, but were only tested (task performance without feedback) once, during the final posttraining test on Day 2. The remaining pre- and posttraining tests were either removed altogether or replaced with stimulus exposure alone.

\section{Analyses}

The dependent variable was the slope of the nonnative category boundary between "mba" and "ba." No learning was expected, or evident on the basis of visual inspection, for the native category boundary between "ba" and "pa," so that boundary slope was not analyzed. The slope of the nonnative category boundary was estimated with a probit function fit to the category responses for each listener and each test separately. Following others (MacKain, Best, and Strange, 1981), the slope values were restricted to the range from 0 to 1 using the formula $0.5-[(\arctan \theta) / \pi]$, where $\theta$ is the slope of the nonnative category boundary. On this scale, 0 indicates a step-wise decrease from $100 \%$ to $0 \%$ "mba" responses as the VOT becomes less negative (phonetically appropriate), .5 indicates a flat slope (no true boundary), and 1 indicates a step-wise increase from $0 \%$ to $100 \%$ "mba" responses (phonetically inappropriate).

The slope values were analyzed using a Bayesian statistical model whose priors yielded conservative estimates for small $N$ (Gelman \& Hill, 2007). This model accounted for bounded values (all values ranged from 0 to 1 ) using a logistic curve. It also accounted for heterogeneous variability across groups using a beta prior over the logistic. The slope value $(y)$ was transformed by $r / 2+y(1-r)$, where $r$ was a relatively small proportion of the data range modeled to best fit the data, in order to increase numerical stability and robustness to outliers. Cauchy distributions with a mean of 0 and a variance of 5 were used as the priors for the model coefficients (Gelman, Jakulin, Pittau, \& Su, 2008). Because the amount of learning depended on the pretraining performance (pretraining test coefficient: $p<.001$ ), performance on the pretraining test on Day 1 served as a covariate. Coefficients for the interaction between group and performance at each test other than the pretraining test on Day 1 were also included. Posterior predictive checks were used to validate these analysis choices. According to these checks, the resulting models accurately predicted the root-mean squared error and the $97.5 \%$, $68.2 \%, 50 \%, 31.8 \%$, and $2.5 \%$ percentiles of the residuals with a standard error of $\leq 0.05$ categorization slope units (posterior predictive $p \geq .41$ ). Model parameters were fitted to the data using the Stan modeling language (version 2.7.0) employing a Markov chain Monte Carlo algorithm (Hoffman \& Gelman, 2014) with four chains of 2,000 iterations, including 1,000 warm-up iterations. Convergence of these four chains to the same solution was verified using the scale reduction statistic (Gelman et al., 2014). In all cases, the chosen number of samples was estimated to yield a prediction error of less than 0.001 categorization slope units. Where appropriate, $p$ values were corrected for multiple comparisons (Gelman, Hill, \& Yajima, 2012; Gelman \& Tuerlinckx, 2000).

\section{Results}

\section{Influence of training amount per day}

To determine the dose-response curve for the amount of training per day with feedback, we trained four groups on the nonnative phonetic classification task, each for a different number of training trials per day (Fig. 1). The group who practiced for 30 trials per day with feedback showed no improvement ( $p=.593$ ), while the groups who practiced for 60 , 120 , or 240 trials per day all showed a steepening of the category boundary slope (all $p \mathrm{~s}<.001$ ) and greater improvement than the 30 -trials/day group ( 30 vs. $60, p=.015$; vs. 120 , $p<.001$; vs. $240, p=.006$ ). The amount of learning did not differ among the groups who practiced 60 or more trials per day, though there was a trend toward greater improvement for 120 than for 240 trials per day ( 60 vs. $>60$ : all $p \mathrm{~s} \geq .126 ; 120$ vs. $240, p=.076$ ). The groups who improved did so between the pre- and posttraining tests on Day 1 and then retained that learning, but did not learn more, on Day 2 (Fig. 2; slopes of regression lines from Post 1 to Post 2: all $p \mathrm{~s} \geq .103$ ). These overall learning outcomes were not affected by the presence or absence of the data from the two listeners in the 120-trial/day group who showed unusually large improvements. However, the across-listener variability was larger for the 120-trial/day group than for the 60 - and 240-trial/day groups when the data from those two listeners were included $(p<.041)$, but not when their data were removed $(p>.130)$. Thus, learning on this nonnative phonetic classification task required between 30 and 60 training trials per day with feedback, 


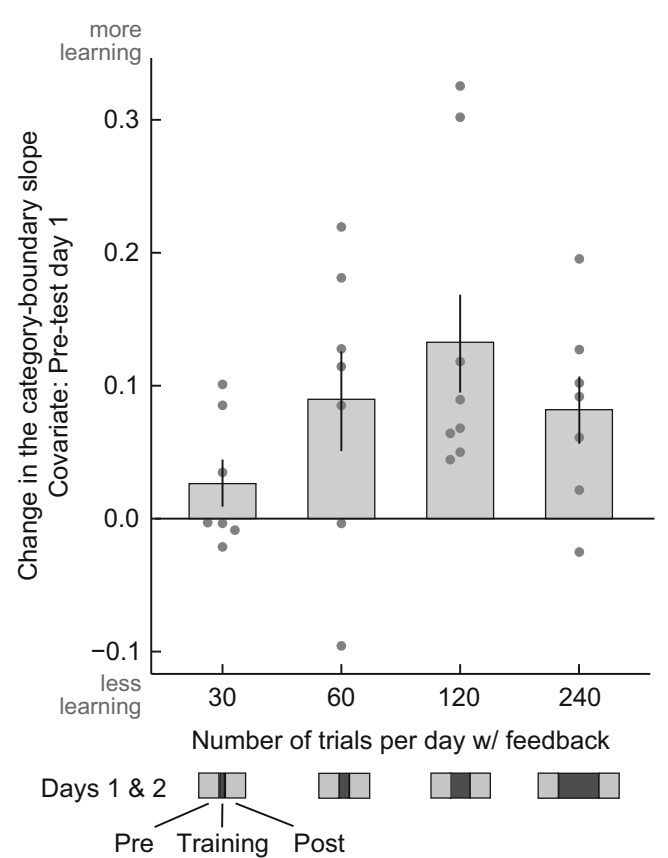

Fig. 1 Amount of improvement: Changes in category boundary slope between "mba" and "ba" from the pretraining test on Day 1 to the posttraining test on Day 2 for individual listeners (gray circles) who practiced the nonnative phonetic classification task for $30(n=7), 60(n$ $=7), 120(n=8)$, or $240(n=7)$ trials per day with feedback ( $x$-axis). Also shown are the means and +1 standard errors for these data (bars and error bars). All values are adjusted using the category boundary slope at the pretraining test on Day 1 as a covariate (Cohen, 1988; see the Method section). Schematic diagrams illustrate the daily regimens: $30,60,120$, or 240 trials per day with feedback (dark gray rectangles) plus 120 -trial preand posttraining tests per day without feedback (light gray rectangles)

additional daily training with feedback beyond that amount did not yield more learning, and all of the improvement occurred on the first day.

\section{Influence of pre- and posttraining tests}

To determine whether the daily 120 -trial pre- and posttraining tests, which included task performance but not feedback, affected learning, we trained another group for 60 trials per day with feedback, but removed all tests but the final one (Fig. 3, diamonds). Performance on the final test for this group was similar to the pretraining performance (triangles at left) and poorer than the final posttraining performance (triangles at right) for the group with 60 trials with feedback and tests (pretraining test Day 1, $p>.999$; posttraining test Day 2, $p=$ $.003)$. The implication is that the testing trials without feedback contributed to the learning shown by the group with tests.

To evaluate whether the contributions from the testing trials resulted from the task performance during testing or simply from the stimulus exposures encountered during testing, we trained a final group for 60 training trials per day with feedback, but replaced all tests but the final one with stimulus exposure alone (Fig. 3, squares). Performance on the final test

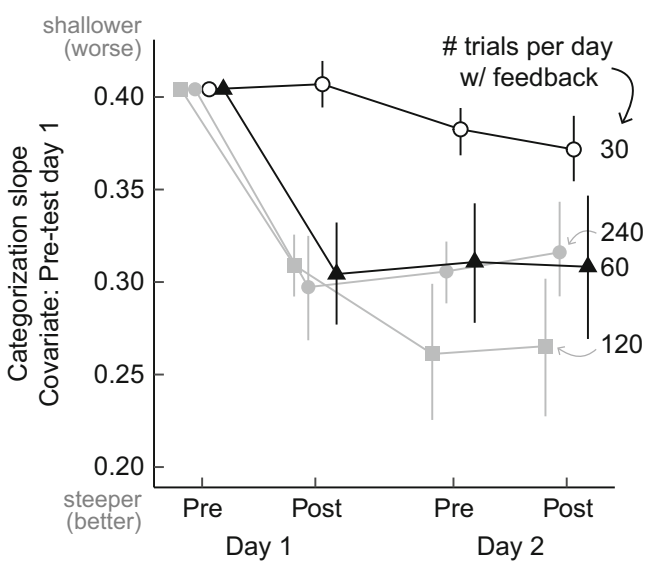

Fig. 2 Learning curves: Means and \pm 1 standard errors of the category boundary slopes between "mba" and "ba" (symbols and error bars) across tests for four different numbers of training trials per day with feedback: 30 (open circles; $n=7$ ), 60 (black triangles; $n=7$ ), 120 (gray squares; $n=8$ ), and 240 (gray circles; $n=7$ ). Values are adjusted using the category boundary slope at the pretraining test as a covariate (Cohen, 1988; see the Method section)

for this group did not differ from that for either the 60-trial group with no tests ( $p=.311$ ) or the 60-trial group with tests ( $p$ $=.213$ ), with the mean falling between the two. However, across-listener variability on the final test was larger for the group with the exposure-only tests than for the group with no tests $(p<.001)$, indicating that the stimulus exposures alone influenced behavior. This pattern mirrored the greater variability for the group with tests, who learned, than for the group with no tests, who did not learn $(p=.002)$. Furthermore, the distributions of final-test performance for individual listeners were similar between the groups with tests and with exposureonly tests. For each of these two groups, the category boundary slope was steeper than the steepest slope for the no-tests group for five listeners, within the range of the slopes for the no-tests group for one listener, and shallower than the shallowest slope of the no-tests group for the remaining one (with tests) or two (exposure-only tests) listeners. The steepest slope was steeper and the shallowest slope shallower for the group with the exposure-only tests than for the group with tests, contributing to the greater overall variability for the group with the exposure-only tests $(p=.010)$.

\section{Discussion}

The present results demonstrate semi-supervised learning on a nonnative phonetic classification task. Performance improved when 60 or more trials with feedback were combined with 120 -trial pre- and posttraining tests without feedback. This learning did not result solely from the 60 trials with feedback, because no learning occurred when only those trials were presented. Likewise, it did not result solely from the 120 trial pre- and posttraining tests without feedback, because 


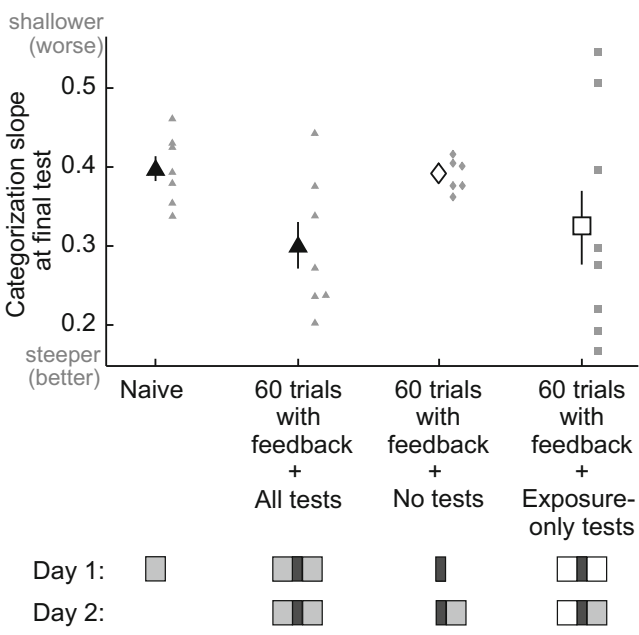

Fig. 3 Means and \pm 1 standard errors of the raw category boundary slopes between "mba" and "ba" at the final test (large symbols and error bars) and the individual data (small symbols) for three groups who practiced 60 trials per day with feedback for two consecutive days with different configurations of pre- and posttraining trials. One group completed 120 -trial pre- and posttraining tests without feedback on both days $(60$ trials with feedback + All tests: triangles, $n=7$, reflecting the data from Figs. 1 and 2; Naïve is the pretraining performance on Day 1 for that group). The second group completed only the final posttraining test on Day 2 (60 trials with feedback + No tests: diamonds, $n=6$ ). The third group also completed only the final posttraining test on Day 2 , but the other tests were replaced with stimulus exposure alone from those tests (60 trials with feedback + Exposure-only tests: squares, $n=8$ ). Schematic diagrams illustrate the regimens: 60 trials per day with feedback (dark gray rectangles), plus various configurations of 120-trial (pre- and posttraining) tests without feedback (light gray rectangles), and 120-trial periods of stimulus exposure alone (open rectangles)

there was no improvement when these tests were combined with 30 trials with feedback or, as we reported previously, when the task performance trials with feedback were replaced with 240 trials of stimulus exposure alone (Wright et al., 2015). If the trials without feedback could generate learning, there should have been learning in both of those cases. These data expand the evidence for semi-supervised learning in humans. In previous reports, trials without feedback led to a shift in a category boundary initially established from trials with feedback (e.g., Kalish et al., 2011; Kalish et al., 2015; Zhu et al., 2007). Here the category boundary was established only by the combination of trials with feedback and trials without feedback. Moreover, these data confirm that semisupervised learning can occur with auditory (new data) as well as with visual (e.g., Kalish et al., 2011; Kalish et al., 2015; Lake \& McClelland, 2011; Rogers et al., 2010; Vong et al., 2016; Zhu et al., 2010; Zhu et al., 2007) stimuli.

The present data also document a steep, saturating, doseresponse curve for the influence of trials with feedback on semi-supervised learning. For the nonnative phonetic classification task examined here, semi-supervised learning was triggered by between 30 and 60 trials of practice with feedback when combined with 240 trials without feedback in the same session. Additional practice with feedback beyond 60 trials did not increase the amount of learning. Thus, it appears that the amount of training with feedback needs to reach some threshold value beyond which semi-supervised learning occurs, and that once this threshold has been reached, additional practice with feedback is superfluous. It is likely that the amount of training with feedback that is required to induce lasting semisupervised learning is task- and stimulus-dependent, on the basis of previous evidence for such dependencies when feedback is provided after every trial (Wright \& Sabin, 2007).

There was also some indication in the present data that semi-supervised learning may not require task performance during the periods without feedback. Given 60 trials with feedback, learning occurred with, but not without, the preand posttraining tests. When the tests were replaced with stimulus exposure alone, mean performance at the final posttraining test fell between that obtained with the tests and that obtained without the tests and did not differ from either of those two cases. However, parallel to the group with tests, across-listener variability was larger for the group with the exposure-only tests than for the no-tests group, indicating that the exposure-only tests affected performance. The distribution of the individual scores for the group with the exposure-only tests was also similar to that for the group with tests, relative to the no-tests group. To the extent that the trials of stimulus exposure alone contributed to learning, the present data provide an unintentional replication of a previous report that learning on this task can be induced or enhanced through a combination of task performance and stimulus exposure alone (Wright et al., 2015). This practice-plus-exposure combination, along with variants of it, has also been shown to aid learning on auditory pure-tone frequency discrimination (Wright, Sabin, Zhang, Marrone, \& Fitzgerald, 2010), visual orientation comparison (Szpiro, Wright, \& Carrasco, 2014), discrimination of musical intervals (Little, Cheng, \& Wright, 2019), and adaptation to foreign accents (Wright et al., 2015). In earlier reports of semi-supervised learning, participants made a response on every trial whether or not feedback was provided. The present results suggest that task performance may be optional for the trials without feedback.

Coming full circle, the present investigation was initially inspired by prior observations that for learning on a finegrained discrimination task to survive across days required sufficient training per day and that additional training beyond that amount was superfluous (e.g., Wright \& Sabin, 2007). The question was whether a similar pattern would also occur for learning on a speech-perception task. The present results do document a learning threshold and improvement plateau that is linked to the amount of daily training (for another example of a learning threshold on a speech task, see Little, Zhang, \& Wright, 2017). However, here the triggering event was a period of sufficient training with feedback per day that catalyzed the benefit from trials without feedback, whereas in 
the previous cases the triggering event had simply been sufficient training per day (within a restricted time period; Little et al., 2017). In addition, once triggered, the improvement here all occurred between the pre- and posttraining tests on the first day, whereas previously the learning had occurred across days. It is not that no additional learning can occur on this task, because listeners who were trained on the same task, but with training alternating between periods of active practice and stimulus exposure alone, ended with better final performance than did the groups trained here (Wright et al., 2015). Notably, when designing the original experiment we had not even considered the possibility that the pre- and posttraining tests without feedback could influence performance, because of previous evidence that learning nonnative phonetic contrasts using constant-stimulus techniques required feedback (McCandliss et al., 2002; Tricomi et al., 2006). The present results replicate the need for feedback for learning on this task, but they show that the feedback is not necessary throughout training.

One practical implication of the present results is that testing periods can affect learning outcomes. When a trained group (who complete pre- and posttesting periods and a training phase) learns more than a control group (who complete only the testing periods) that additional improvement is typically attributed to the training phase. The present data illustrate that this outcome can also arise from an interaction between the training phase and testing periods. Another implication is that speech learning that is fostered by the combination of periods of task practice with periods of stimulus exposure alone (Wright et al., 2015) can occur with only a relatively small number of trials with feedback. Use of this combination training could therefore markedly reduce the need for direct task practice during speech training.

\section{Summary}

1. For a nonnative phonetic classification task, practice with feedback for 30 trials/day yielded no learning, while practice for 60,120 , or 240 trials/day led to similar steepenings of the category boundary slope (Figs. 1 and 2).

2. Removing the 120-trial daily pre- and posttraining tests without feedback halted learning (Fig. 3), indicating that the tests without feedback contributed to the improvement.

3. Replacing the pre- and posttraining tests with stimulus exposure alone largely restored the performance obtained with the tests (Fig. 3), suggesting that the necessary component for learning that was supplied by the tests was the stimulus exposures rather than the task performance that they provided.

4. Together these results suggest that training with feedback can function as an all-or-none trigger for recruiting the contribution of trials without feedback, or possibly mere stimulus exposures, to induce semi-supervised learning on a speech task.
Author note This work was sponsored in part by the Defense Advanced Research Projects Agency (DARPA) Biological Technologies Office (BTO) TNT program, under the auspices of Doug Weber and Tristan McClure-Begley, through the Space and Naval Warfare Systems Center, Pacific Grant/ Contract No. N66001-17-2-4011, and by the Knowles Hearing Center at Northwestern University. Any opinions, findings, and conclusions or recommendations expressed in this publication are those of the authors and do not necessarily reflect the views of the DARPA BTO. The data and analyses for all experiments are available at https://osf.io/d83c4/. None of the experiments was preregistered.

\section{References}

Aberg, K. C., Tartaglia, E. M., \& Herzog, M. H. (2009). Perceptual learning with chevrons requires a minimal number of trials, transfers to untrained directions, but does not require sleep. Vision Research, 49, 2087-2094. https://doi.org/10.1016/j.visres.2009.05.020.

Boersma, P. (2001). Praat, a system for doing phonetics by computer. Glot International, 5, 341-345.

Cohen, J. (1988). The concepts of power analysis. In Statistical power analysis for the behavioral sciences (pp. 1-17). Hillsdale, NJ: Erlbaum.

Gelman, A., Carlin, J. B., Stern, H. S., Dunson, D. B., Vehtari, A., \& Rubin, D. B. (2014). Bayesian data analysis (3rd ed.). Boca Raton, FL: Chapman \& Hall/CRC.

Gelman, A., \& Hill, J. (2007). Data analysis using regression and multilevel/hierarchical models. Cambridge, UK: Cambridge University Press.

Gelman, A., Hill, J., \& Yajima, M. (2012). Why we (usually) don't have to worry about multiple comparisons. Journal of Research on Educational Effectiveness, 5, 189-211.

Gelman, A., Jakulin, A., Pittau, M. G., \& Su, Y.-S. (2008). A weakly informative default prior distribution for logistic and other regression models. Annals of Applied Statistics, 2, 1360-1383. https://doi. org/10.2139/ssrn.1010421.

Gelman, A., \& Tuerlinckx, F. (2000). Type S error rates for classical and Bayesian single and multiple comparison procedures. Computational Statistics, 15, 373-390.

Gibson, B. R., Rogers, T. T., \& Zhu, X. (2013). Human semi-supervised learning. Topics in Cognitive Science, 5, 132-172. doi: https://doi. org/10.1111/tops.12010.

Hauptmann, B., \& Karni, A. (2002). From primed to learn: the saturation of repetition priming and the induction of long-term memory. Cognitive Brain Research, 13, 313-322.

Hauptmann, B., Reinhart, E., Brandt, S.A., \& Karni, A. (2005). The predictive value of the leveling off of within session performance for procedural memory consolidation. Cognitive Brain Research, 24, 181-189.

Hoffman, M. D., \& Gelman, A. (2014). The no-U-turn sampler: Adaptively setting path lengths in Hamiltonian Monte Carlo. Journal of Machine Learning Research, 15, 1593-1623.

Kalish, C. W., Rogers, T. T., Lang, J., \& Zhu, X. (2011). Can semisupervised learning explain incorrect beliefs about categories? Cognition, 120, 106-118. https://doi.org/10.1016/j.cognition.2011. 03.002.

Kalish, C. W., Zhu, X., \& Rogers, T. T. (2015). Drift in children's categories: when experienced distributions conflict with prior learning. 
Developmental Science, 18, 940-956. https://doi.org/10.1111/desc. 12280.

Lake, B. M., \& McClelland, J. L. (2011). Estimating the strength of unlabeled information during semi-supervised learning. Proceedings of the Annual Meeting of the Cognitive Science Society, 33. Retrievedfrom https://escholarship.org/uc/item/ $4 \mathrm{pt37633.}$

Little, D. F., Cheng, H. H., \& Wright, B. A. (2019). Inducing musicalinterval learning by combining task practice with periods of stimulus exposure alone. Attention, Perception, \& Psychophysics, 81, 344 357. https://doi.org/10.3758/s13414-018-1584-x .

Little, D. F., Zhang, Y.-X., \& Wright, B. A. (2017). Disruption of perceptual learning by a brief practice break. Current Biology, 27, 36993705. https://doi.org/10.1016/j.cub.2017.10.032 .

MacKain, K. S., Best, C. T., \& Strange, W. (1981). Categorical perception of English /r/ and /1/ by Japanese bilinguals. Applied Psycholinguistics, 2, 369-390.

McCandliss, B. D., Fiez, J. A., Protopapas, A., Conway, M., \& McClelland, J. L. (2002). Success and failure in teaching the [r]-[1] contrast to Japanese adults: Predictions of a Hebbian model of plasticity and stabilization in spoken language perception. Cognitive, Affective, \& Behavioral Neuroscience, 2, 89-108. https://doi.org/10.3758/CABN.2.2.89.

McClaskey, C. L., Pisoni, D. B., \& Carrell, T. D. (1983). Transfer of training of a new linguistic contrast in voicing. Perception \& Psychophysics, 34, 323-330.

Ofen-Noy, N., Dudai, Y., \& Karni, A. (2003). Skill learning in mirror reading: how repetition determines acquisition. Cognitive Brain Research, 17, 507-521.

Ortiz, J. A., \& Wright, B. A. (2010). Differential rates of consolidation of conceptual and stimulus learning following training on an auditory skill. Experimental Brain Research, 201, 441-451. https://doi.org/ 10.1007/s00221-009-2053-5.

Pisoni, D. B., Aslin, R. N., Perey, A. J., \& Hennessy, B. L. (1982). Some effects of laboratory training on identification and discrimination of voicing contrasts in stop consonants. Journal of Experimental Psychology: Human Perception and Performance, 8, 297-314. https://doi.org/10.1037/0096-1523.8.2.297 .

Rogers, T. T., Kalish, C. W., Gibson, B. R., Harrison, J., \& Zhu, X. (2010). Semi-supervised learning is observed in a speeded but not an unspeeded 2D categorization task. Proceedings of the Annual Meeting of the Cognitive Science Society, 32. Retrieved from https://escholarship.org/uc/item/8g54r9cm.

Savion-Lemieux, T., \& Penhune, V. B. (2005). The effects of practice and delay on motor skill learning and retention. Experimental Brain Research, 161, 423-431.
Szpiro, S. F., Wright, B. A., \& Carrasco, M. (2014). Learning one task by interleaving practice with another task. Vision Research, 101, 118 124. https://doi.org/10.1016/j.visres.2014.06.004 .

Tremblay, K., Kraus, N., Carrell, T. D., \& McGee, T. (1997). Central auditory system plasticity: Generalization to novel stimuli following listening training. Journal of the Acoustical Society of America, 102, 3762-3773.

Tricomi, E., Delgado, M. R., McCandliss, B. D., McClelland, J. L., \& Fiez, J. A. (2006). Performance feedback drives caudate activation in a phonological learning task. Journal of Cognitive Neuroscience, 18, 1029-1043. https://doi.org/10.1162/jocn.2006.18.6.1029 .

Vong, W. K., Navarro, D. J., \& Perfors, A. (2016). The helpfulness of category labels in semi-supervised learning depends on category structure. Psychonomic Bulletin \& Review, 23, 230-238. doi: https://doi.org/10.3758/s13423-015-0857-9.

Wright, B. A., Baese-Berk, M. M., Marrone, N., \& Bradlow, A. R. (2015). Enhancing speech learning by combining task practice with periods of stimulus exposure without practice. Journal of the Acoustical Society of America, 138, 928-937. https://doi.org/10. 1121/1.4927411 .

Wright, B. A., \& Sabin, A. T. (2007). Perceptual learning: How much daily training is enough? Experimental Brain Research, 180, 727 736.

Wright, B. A., Sabin, A. T., Zhang, Y., Marrone, N., \& Fitzgerald, M. B. (2010). Enhancing perceptual learning by combining practice with periods of additional sensory stimulation. Journal of Neuroscience, 30, 12868-12877. https://doi.org/10.1523/JNEUROSCI.0487-10. 2010.

Zhu, X., Gibson, B. R., Jun, K.-S., Rogers, T. T., Harrison, J., \& Kalish, C. (2010). Cognitive models of test-item effects in human category learning. In J. Fürnkranz \& T. Joachims (Eds.), Proceedings of the 27th International Conference on Machine Learning (pp. 12471254). Madison, WI: Omnipress.

Zhu, X., \& Goldberg, A. B. (2009). Introduction to semi-supervised learning. San Rafael, CA: Morgan \& Claypool.

Zhu, X., Rogers, T. T., Qian, R., \& Kalish, C. W. (2007). Humans perform semi-supervised learning too. Paper presented at the TwentySecond AAAI Conference on Artificial Intelligence (AAAI-07), Vancouver, BC.

Publisher's note Springer Nature remains neutral with regard to jurisdictional claims in published maps and institutional affiliations. 